\title{
RECENT DEVELOPMENTS IN THE UNDERSTANDING OF FRETTING FATIGUE
}

\author{
D. Nowell \\ Department of Engineering Science, University of Oxford \\ Parks Road, Oxford, OX1 3PJ, UK \\ david.nowell@eng.ox.ac.uk
}

\begin{abstract}
Considerable progress has been made in the understanding of fretting fatigue over the last decade. Experiments have become more standardised and carefully controlled and this has provided the data necessary for development of methods for predicting fretting fatigue performance. This paper reviews a number of recent developments, starting with attempts to apply multiaxial initiation criteria to the fretting problem. The importance of the size effect is highlighted and an analogy is made between fretting and notch fatigue. Methods for characterising crack initiation using asymptotic analysis are discussed, together with short crack arrest concepts which provide a means of predicting fretting fatigue limits from plain fatigue data.
\end{abstract}

\section{Introduction}

The phenomenon of 'fretting' has been recognised and studied for well over a century. Initially it was recognised primarily as a surface damage phenomenon; essentially what might today be called 'fretting wear'. The first recorded reference in the literature is in a paper by Eden et al. [1], published in 1911. These authors report the presence of fretting debris, interpreted as iron oxide, in the neighbourhood of a contact interface. Although fretting fatigue is not specifically mentioned, the contact in question was that between grips and specimen in a plain fatigue test. In the years since then, there must have been many researchers who have suffered unintended fretting fatigue failures in this configuration. Indeed, fretting between a specimen and grips has been used the basis of a fretting fatigue test by Hutson et al. [2]. Following the early investigations, fretting was recognised as being associated with a reduction in fatigue life (Tomlinson et al, [3], Warlow-Davies [4]) and the term 'fretting fatigue' came into common use to describe situations where microslip between contacting surfaces appears to give rise to a reduction in fatigue life when compared to a plain component.

Fretting fatigue has been responsible for a large number of service failures across a wide range of applications. For example, fretting in railway axles was reported by Maxwell et al. [5] in 1967, yet remains a cause for concern over thirty years later [6]. For obvious reasons, fretting fatigue is particularly important in safety-critical industries such as aerospace or nuclear power generation. The recent High Cycle Fatigue (HCF) initiative [7] in the USA has provided a focus for fretting research in the aerospace sector, particularly in aircraft engine applications. Here there are a number of important contact features which may suffer fretting fatigue. These include the 'dovetail' roots of compressor blades (Nowell, [8]), where failure may have serious consequences for engine integrity (Xi et al., [9]).

Experimental investigations of fretting fatigue have taken a number of forms. Initially it was common to use 'bridge' type fretting pads, as shown in Fig. 1a (e.g. Fenner and Field, 
[10]) and these remained popular until the early 1990s. The chief virtue is simplicity in that a normal fatigue specimen may be used, either in bending or cyclic tension. The bridges are simply clamped to the sides of the specimen by a proving ring or similar arrangement and the cyclic strain in the specimen causes relative motion between the bridge feet and the specimen. Unfortunately, there are a number of difficulties with such a simple arrangement. Contact conditions at the pad feet are difficult to characterize, particularly if there is bending in the bridge itself. Further, conditions at each foot will not be absolutely identical and it is likely that one foot will slip before the other, even under nominally symmetric conditions. This means that the slip regime during the experiment is often unknown.

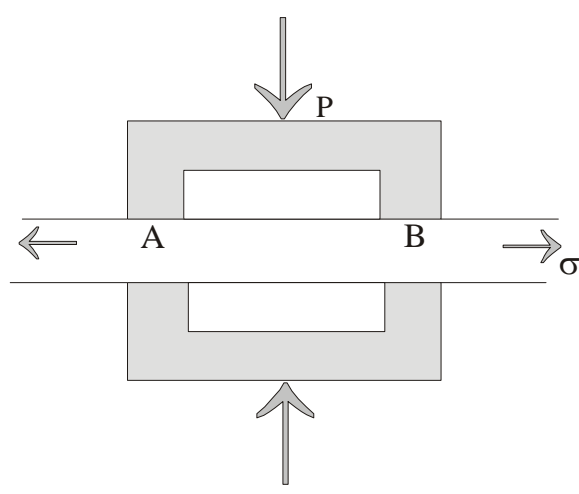

(a)

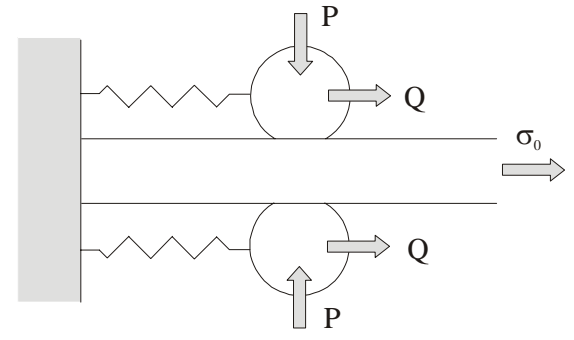

(b)

FIGURE 1. Geometry of fretting fatigue experiments: (a) bridge-type pads; (b) cylindrical pads

A landmark series of papers were published in the late 1960s and early 1970s by Nishioka and Hirakawa (e.g. [11]). These used a quite different contact configuration of cylindrical pads clamped against a flat specimen (Fig. 1b). This geometry has a number of advantages: pad alignment is less critical and the stresses may, in principle, be predicted by classical contact analysis (Hertz [12], Mindlin [13]). Further, the important parameters for subsequent stress analysis (normal load, P, tangential load $\mathrm{Q}(\mathrm{t})$, and specimen remote stress $\sigma(\mathrm{t})$ may all be readily measured and controlled. The geometry has since been adopted by a large number of other researchers, including Bramhall [14], Hills et al. [15], and Szolwinski and Farris [16]. In this type of test the normal load is normally fixed, wheras the tangential load is cycled, and applied using springs (Fig. 1b) or a separate actuator (Mugadu et al. [17]).

Other types of experiment may be more closely related to the geometry of the actual component. For the dovetail geometry, such experiments have been carried out by Ruiz et al. [18], Papaniknos and Meguid, [19], and Conner and Nicholas [20]. A development of Ruiz's apparatus is still in use at Oxford and is shown schematically in Fig. 2. In this implementation, blade loads, representing centrifugal force in the engine are applied to two opposing blade specimens. These are mounted in a central disk specimen, which is also subject to load, simulating disk expansion under centrifugal loading. This is an important feature, since it allows accurate representation of relative slip in the engine, which may be important for wear of coatings. High cycle fatigue loads, representing blade vibration are applied by mechanical shaker units clamped to each blade. Figure 3. shows a photograph of a failed dovetail specimen from this rig. The origin of the fatigue crack at the edge of the contact flank may be clearly seen. 


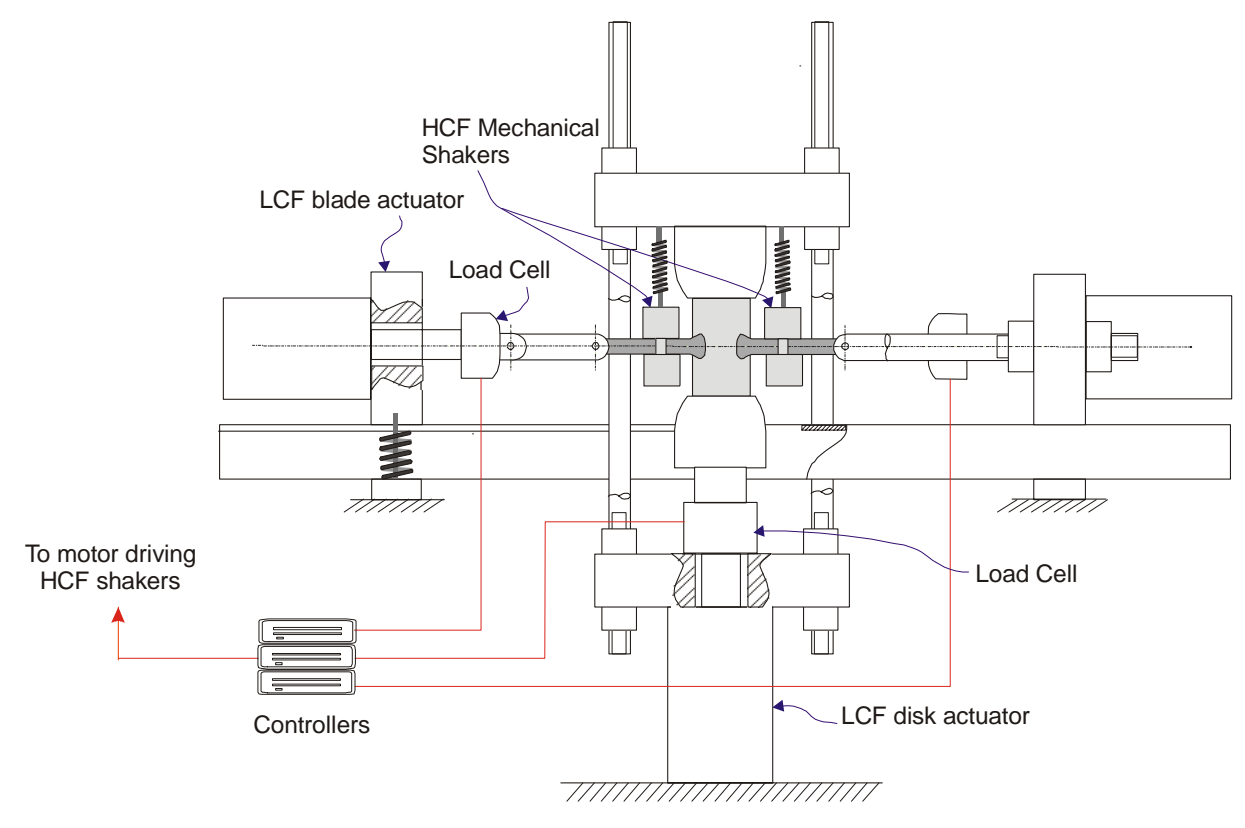

FIGURE 2. Schematic diagram of biaxial dovetail fretting fatigue apparatus.

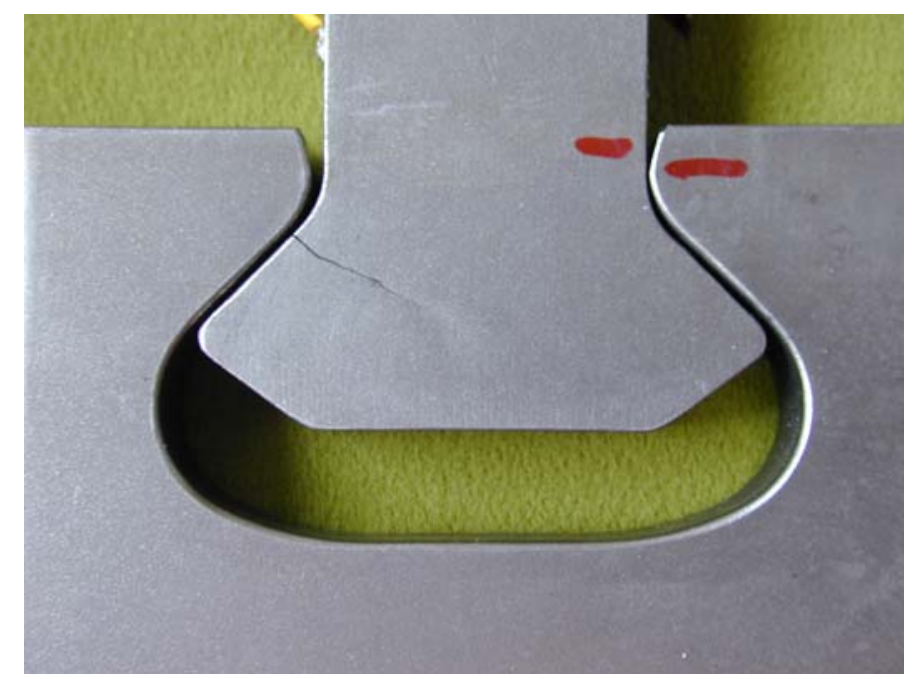

FIGURE 3. Failed specimen from biaxial dovetail rig.

\section{Features of fretting fatigue}

Relative to plain fatigue, fretting fatigue displays a number of important features which must be considered in any analysis of experimental results or design situations:

i. Stress gradients are likely to be very high, due to the localised stress concentration at the contact. The magnitude of these stress gradients is usually much higher than those associated with typical design features of components (e.g. notches and holes)

ii. Loading is likely to be non-proportional in the neighbourhood of the contact, even if the external loads are applied in a proportional fashion. This feature is caused by the non-linear nature of the friction at the contact interface.

iii. Initiated cracks will experience a variable R-ratio as they grow away from the contact. 
iv. Localised surface damage at the asperity level may play a role in accelerating the initiation of cracks at the asperity scale.

Feature (i) is particularly important since it is likely to lead to a much stronger size effect in fretting fatigue than in plain fatigue. Because of the severity of the stress gradient this effect is likely to be even more significant than that in notch fatigue. Bramhall [14] was the first to investigate the size effect in a systematic way by noting that the peak pressure, $p_{0}$, in a cylindrical Hertzian contact is related to the normal load, $P$, and the pad radius, $R$, by

$$
p_{0}=\sqrt{\frac{P E^{*}}{\pi R}}
$$

where $E^{*}$ is a constant for the material pair (Johnson [21]). The semi-width of the contact, $a$, is given by

$$
a=\sqrt{\frac{4 P R}{\pi E^{*}}}
$$

Hence the $p_{0}$ is proportional to $\sqrt{ }(P / R)$, whereas $a$ is proportional to $\sqrt{ }(P R)$. It is therefore possible to vary the extent of the contact and its associated stress field (measured by $a$ ) whilst keeping the magnitude of the stresses constant. In practice, the situation is a little more complicated than this, since the finite thickness of the specimen can affect the subsurface stress field (Fellows et al. [22], Hills and Nowell [23]). However, experiments show that there is a clear variation in fatigue life with contact size [14], [15]. Figure 4 presents typical data for an Al-4Cu alloy [24].

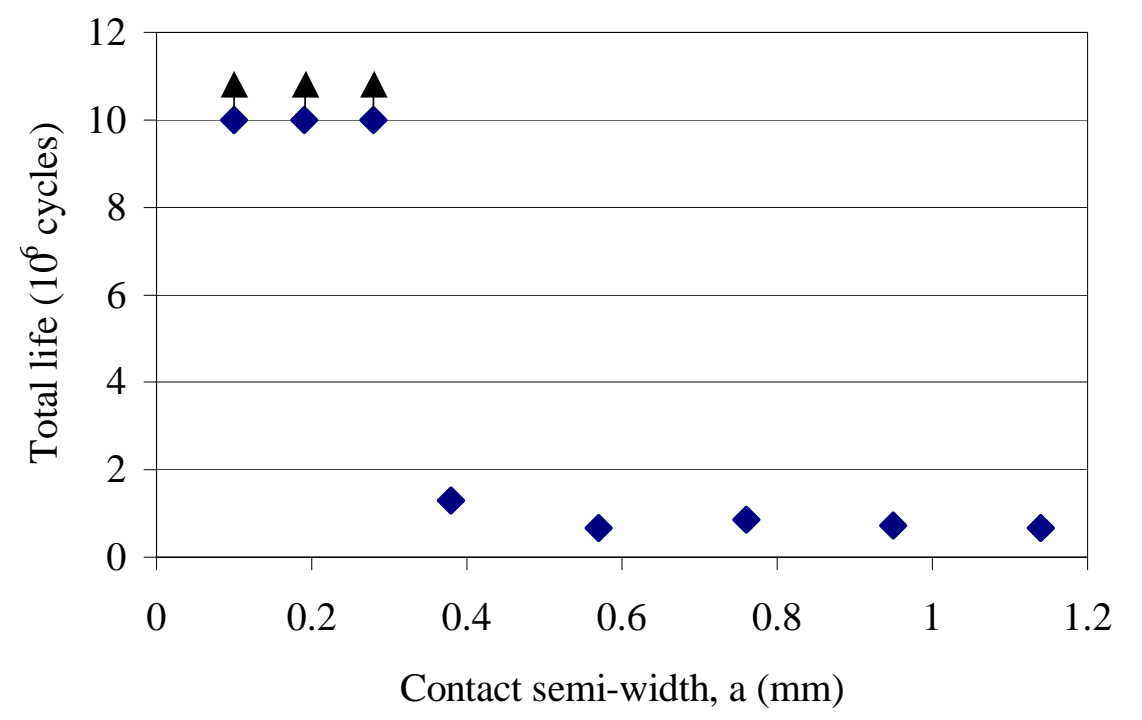

FIGURE 4. Typical fretting fatigue results for an Al-4Cu alloy, showing the size effect

Feature (iv), the role of surface damage has been a matter of debate. Many authors have reported a variation in fatigue life with slip amplitude (e.g. Nishioka and Hirakawa [25]). Broadly, fatigue life is often found to decrease with increasing slip amplitude up to a certain threshold value (typically $50 \mu \mathrm{m}$ ), then increase again. This effect can be summarised in the diagram shown in Fig.5, first suggested by Vingsbo and Söderberg [26]. Much of the experimental evidence, however, has been obtained under conditions of partial slip, where a variation in slip amplitude may equally well be interpreted as a variation in tangential force. 
This might explain the initial decrease in fatigue life with increasing slip. The increase for slip amplitudes greater than $50 \mu \mathrm{m}$ is often ascribed to the rapid increase in wear rate, which 'wears away' embryo cracks before they can propagate. Another possibility is that the wear debris itself forms a solid lubricant layer, thereby reducing stresses (Waterhouse [27]). Ciavarella et al. have recently pointed out [28] that it is not always straightforward to separate out surface damage and stress effects and this may be one reason why the debate is still running.

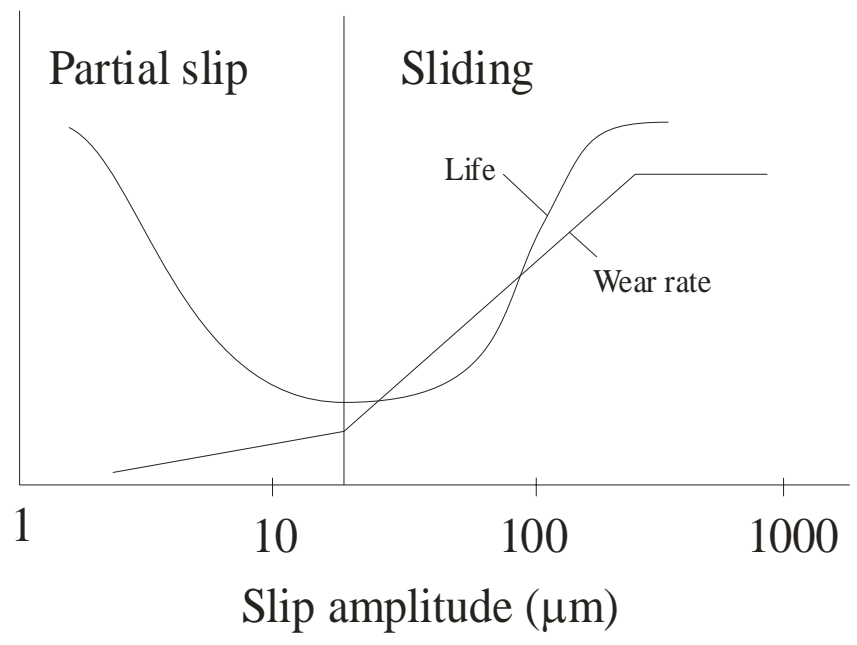

FIGURE 5. Schematic diagram of the variation of fretting fatigue life and fretting wear rate with slip amplitude (after [26]).

\section{Prediction of fretting fatigue performance}

Early attempts at the prediction of fretting fatigue performance frequently employed 'special' empirical parameters, formulated purely for the fretting case. These were perhaps thought necessary because of the features of fretting fatigue mentioned earlier which caused difficulties in applying standard fatigue parameters. Particularly popular were the parameters proposed by Ruiz [18]. The first of these was an energy based damage parameter $(\tau \delta)$ formed from the product of the local slip amplitude with the maximum shear traction. A second, enhanced parameter $(\sigma \tau \delta)$ was proposed by multiplying this by the maximum local stress component parallel to the contact surface. This was found to give a better estimation of the location of fretting crack initiation, although the physical interpretation of the parameter is much less clear. It seems unlikely, for example, that a critical value of either of Ruiz's parameters could be regarded as a material constant. Nevertheless, such parameters often provide an acceptable means of correlating across a range of experimental conditions for a given pair of contacting materials [28].

Attempts to apply more general fatigue parameters to the fretting problem have often started by recognising the inherent multiaxial nature of the stress field. Neu et al. [29] applied a range of parameters, including the Fatemi-Socie [30] and Smith, Watson, Topper [31] approaches to the interpretation of tests carried out on PH 13-8 stainless steel. They found that the F-S parameter was most effective at prediction the location and initial growth direction of fatigue cracks. The FS parameter is based on shear initiation along critical planes and may be defined as

$$
\Gamma_{F S}=\frac{\Delta \gamma}{2}\left\{1+\frac{\alpha \sigma_{\max }}{\sigma_{y}}\right\}
$$


where $\Delta \gamma$ is the shear strain range on the critical plane, $\sigma_{\max }$ is the maximum value of direct stress across the plane, $\sigma_{\mathrm{y}}$ is the yield stress, and $\alpha$ is a fitting parameter. No attempt was made in [29] to correlate the parameters with initiation life. However, the same pair of critical plane parameters were also used in the analysis of experiments in $\mathrm{Al}-4 \mathrm{Cu}$ and Ti-6Al-4V by Araújo and Nowell [32]. Here, initiation life was predicted and was shown to correlate reasonably well with Nowell's results ([24], presented here in Fig. 4) for the large contacts in each series. However, since the magnitude of the stress was the same for all contact sizes, the increase in life at lower values of $a$ could not be predicted by the straightforward application of the method. To overcome this difficulty, averaging of the parameters (or the underlying stress components) over a critical volume was proposed and results are presented in Fig. 6. Averaging of the FS parameter is carried out over a cubic volume of side $d$. It will be seen that a value of $d$ between 50 and $80 \mu \mathrm{m}$ appears to give the best fit to the experimental results. This value is similar to a typical microstructural dimension for the material, which has an elongated grain structure with a grain diameter of the order of $100 \mu \mathrm{m}$.

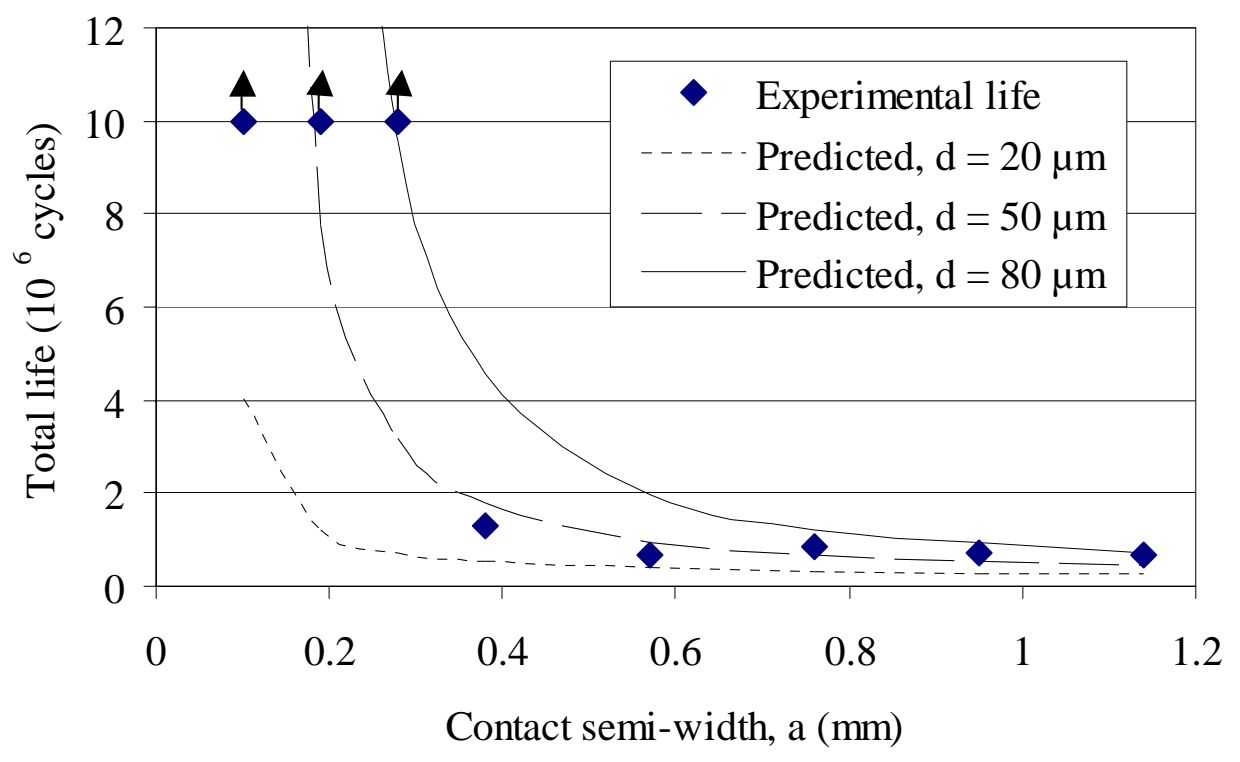

FIGURE 6. Comparison of experimental lives with predicted values for different averaging volumes for experiments with $\mathrm{Al}-4 \mathrm{Cu}$ [24].

Other authors have applied different multiaxial parameters to crack initiation in fretting. Fouvry et al. [33] have applied Dang Van’s mesoscopic parameter (Dang Van et al. [34]) and have shown that an averaging dimension of $6 \mu \mathrm{m}$ is required in a low alloy steel (30NCD16) in order that the fretting results are consistent with those for plain fatigue in the same material. It should be noted that, whilst Dang Van's parameter starts from a physical basis in considering the shakendown stress state within an individual grain, a number of approximations are required in order to use it with macroscopically observable material parameters. Peridas et al. [35] have shown that the parameter is in fact very similar to a number of classical bulk parameters, including the Goodman and Sines relationships. In an alternative approach, Murthy et al. [36] have applied a modified Manson-McKnight multiaxial model which also avoids the requirement to calculate critical planes. Once again, some form of correction was found to be required in order to account for the high stress gradients present. Here a weakest link approach was followed, rather than volume averaging. 


\section{Notch analogies}

As discussed above, it would appear that the stress state under a typical frictional contact is highly multiaxial and undergoes non-proportional loading. This is true for the case of a general point, but it is frequently the case that the most highly loaded point is at or near the edge of contact. This can be clearly seen in Fig. 7, which shows the SWT and FS parameters for the load case corresponding to the experimental results in Fig. 4. If the most highly stressed point is at the contact edge and the contact is 'incomplete' [21], the normal and shear tractions will have fallen to zero at this point and hence the only non-zero stress component at the surface will be that parallel to the surface. Hence the stress state at the point of initiation is likely to be uniaxial (or very close to it). This means that the complication of using multiaxial parameters might reasonably be avoided in many circumstances. With this approximation, it is possible to draw an analogy between the stress state at the contact edge and that in a suitably shaped notch (Fig. 8). This is a rather loose analogy, since it is restricted to matching stresses along a line in each geometry (the notch bisector and the surface normal at the edge of contact). However, good results can be achieved by varying the notch size, root radius, opening angle, and remote load. Figure 9 shows some example results, comparing stresses beneath a 'flat and rounded' contact with those in the neighbourhood of a notch.

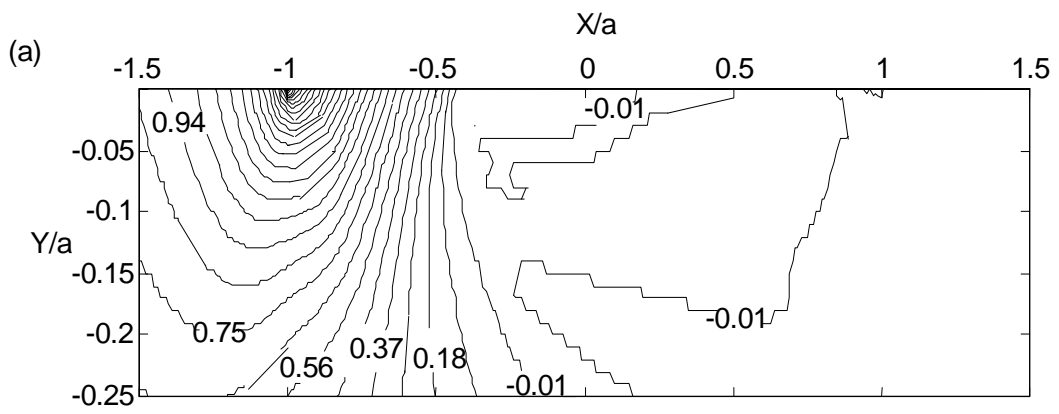

(b)

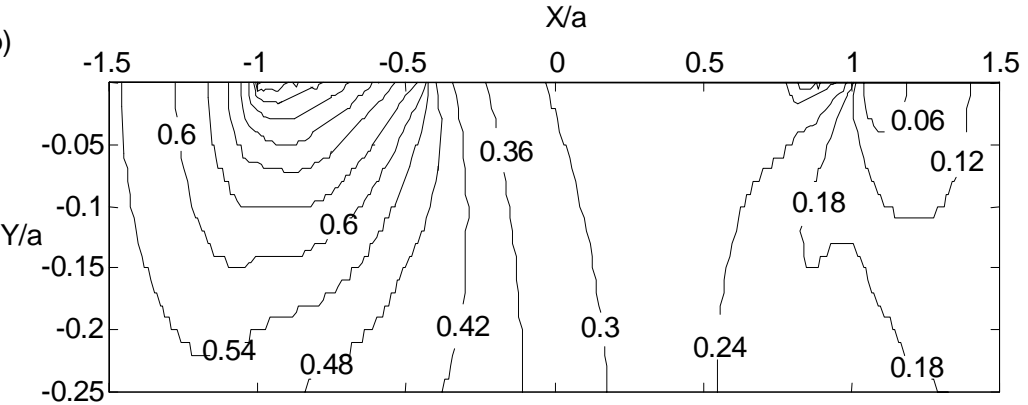

FIGURE 7. Contours of (a) SWT parameter $\left(\Gamma_{\mathrm{SWT}} \mathrm{E} / \mathrm{p}_{0}{ }^{2}\right)$ and (b) FS parameter $\left(\Gamma_{\mathrm{FS}} \mathrm{G} / \mathrm{p}_{0}\right)$ corresponding to the experimental results in Fig.4.

This analogy suggests that it might be possible to apply traditional notch fatigue life prediction approaches, such as the 'point', 'line', and 'area' methods (Taylor [38]), to the interpretation of fretting fatigue. The approach certainly has its uses, notably in reading across from experiments at different contact conditions in the same experimental series, but a degree of empiricism is required in choosing the critical distance. The high stress gradients present, when compared to notches introduced as design features, mean that it is unlikely that standard values can be used. An example of this application is shown in Fig. 10, which predicts the 'fatigue limit' for a set of fretting fatigue experiments as a function of contact 
size (specifically, since this is a flat and rounded configuration, the size of the stress concentration in the rounded zone at the edge of contact).
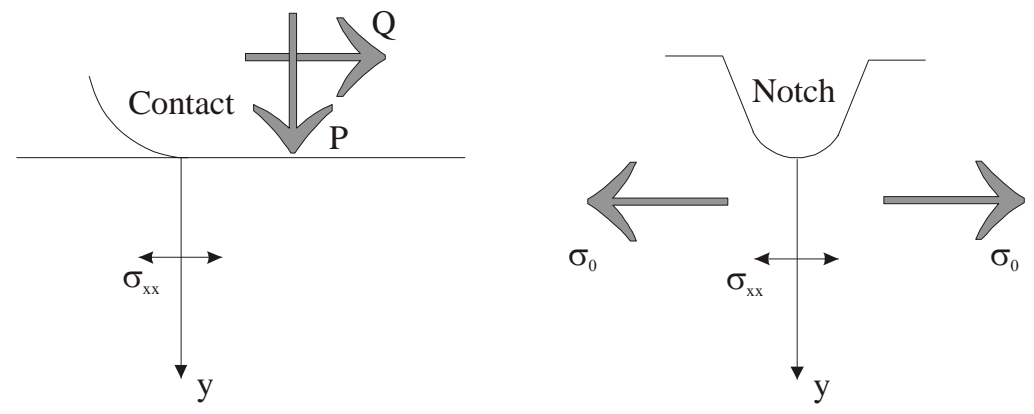

FIGURE 8. Analogy between stresses at a contact and at a notch.

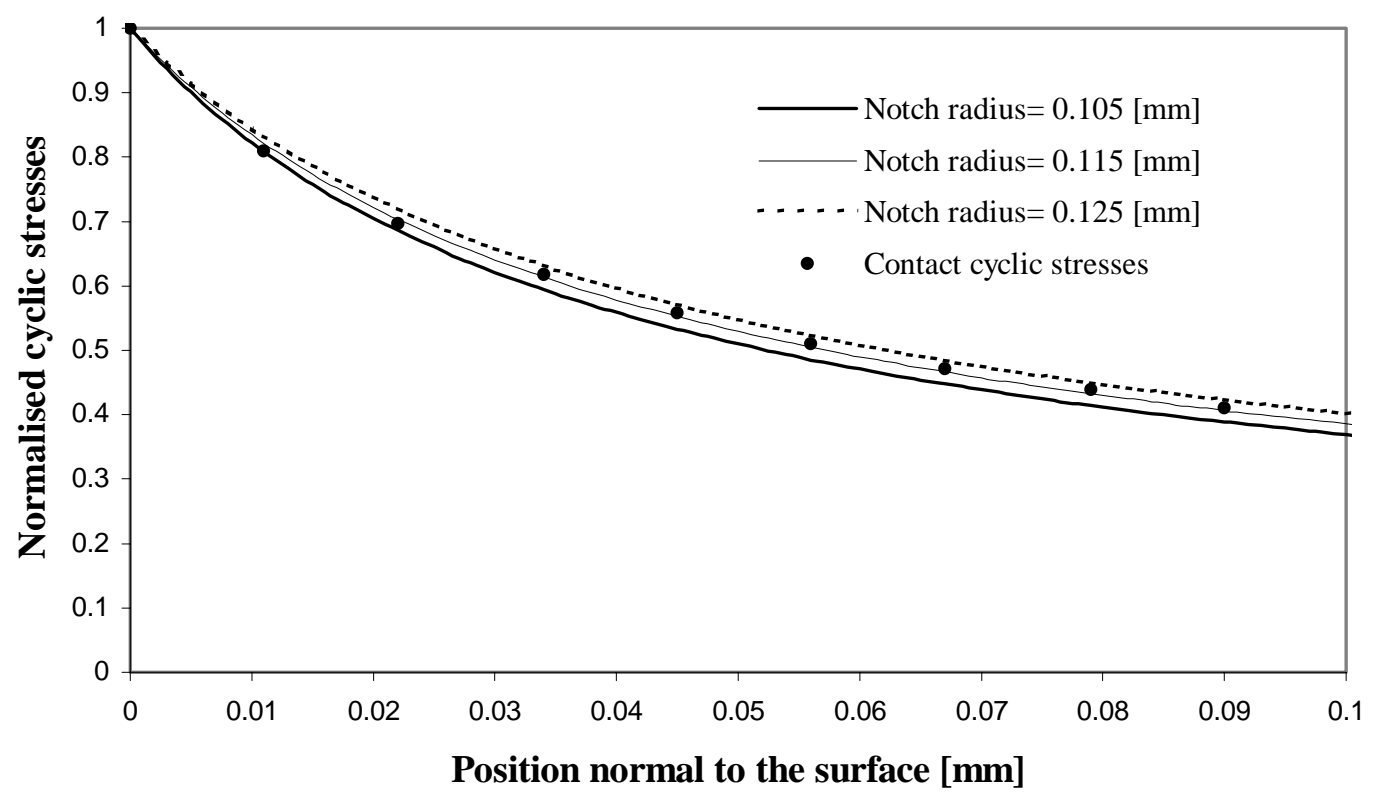

FIGURE 9. Comparison between notch and cyclic stresses (from Nowell and Dini [37]).

\section{Crack analogies and asymptotic approaches}

Over the last decade, an new means of assessing stresses at contacts has developed, based on asymptotic analysis. Giannakopoulos et al. [39] noted that there are some parallels between the stress field close to the edge of a flat rigid punch and that at the tip of an elastic crack. In particular, the pressure distribution for a flat rigid punch (with sharp corners) resting on a half-plane is given by

$$
p(x)=\frac{P}{\sqrt{a^{2}-x^{2}}}
$$

where $P$ is the normal load (per unit length) and $2 a$ the width of the punch. It will be apparent that there are singularities in pressure at the edge of the punch and these may be investigated further by taking an asymptotic expansion of (4) using a co-ordinate $r=x+a$. This gives (for the left hand edge) 


$$
p(x)=-\sigma_{y y} \rightarrow \frac{P}{\pi \sqrt{2 a r}}
$$

The analogy with the stresses at the tip of a crack will be clearly seen. Tangential loading of the punch may be treated in a similar way and is found to be analogous to mode II loading of a crack. In practice, of course, the high stresses present at the edge of contact are likely to be relieved by material yield. This consideration leads to an equivalent restriction to that of 'small scale yielding' applied to crack problems. Since the loading produced by the punch is analogous to mode I and II loading of a crack, it may be seen that fretting fatigue crack growth for this geometry is related to the problem of a branch crack growing from the tip of a primary elastic crack [39].

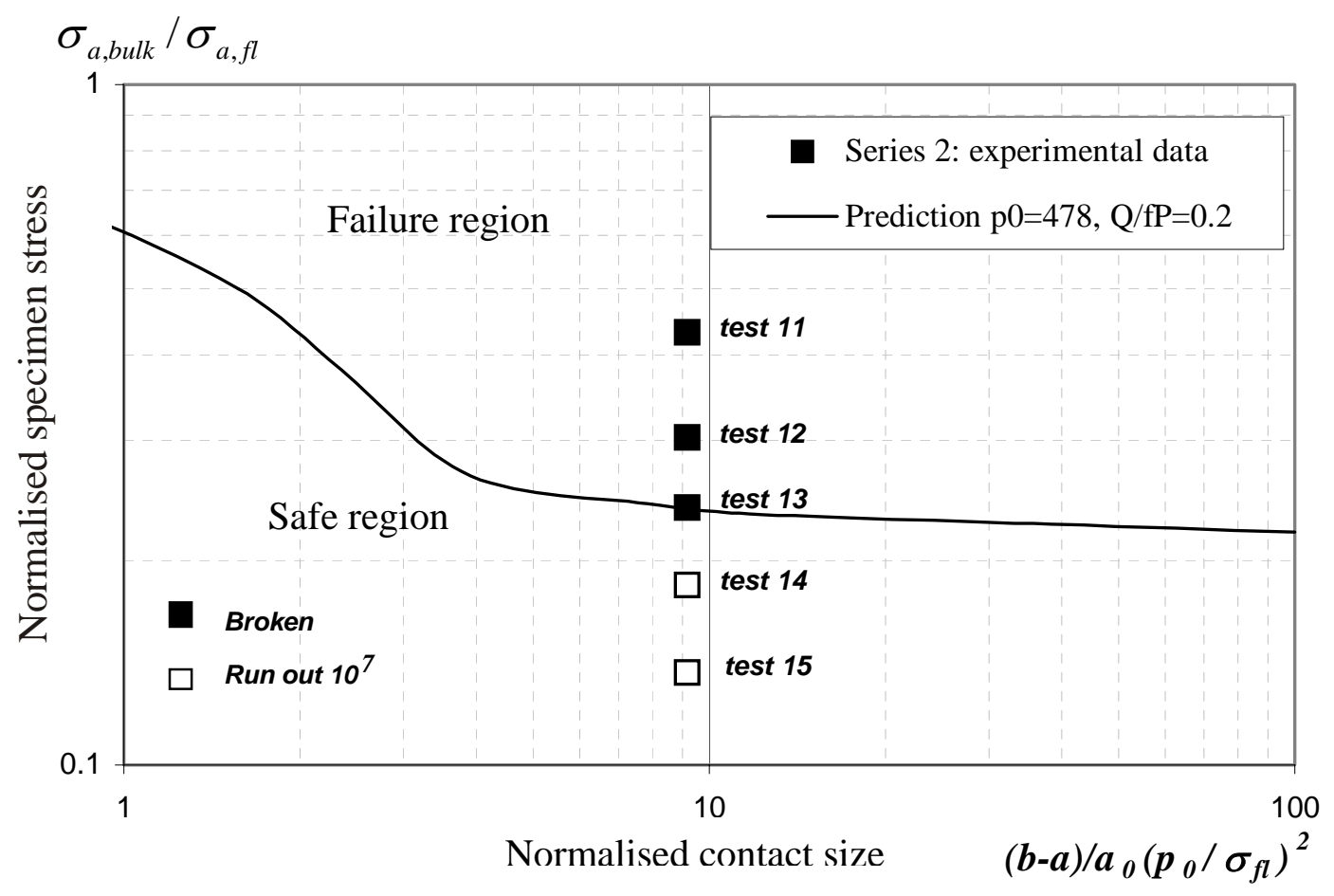

FIGURE 10. Example of the use of the notch analogy to predict fretting fatigue performance: fatigue strength as a function of contact size, compared to experimental results for Ti-6Al-4V.

The original results of Giannakopoulos et al. are rather restrictive in terms of geometry and the application to practical fretting fatigue situations is therefore not entirely straightforward. However the original idea has been extended to the case of a flat punch with radiused corners (the so-called 'flat and rounded' contact), Giannakopoulos et al. [40]. In this case the analogy is with a blunt notch rather than with a crack. Ciavarella [41] has extended the approach still further in formulating the 'Crack like notch analogue', or CLNA model. He notes that the size effect means that a fretting contact can effectively be classified into three distinct regions, in a similar manner to that proposed for notches by Atzori and Lazarin [42]: (i) cases where the stress concentration affects such a small area that it may be ignored, (ii) cases of intermediate size where the concentration behaves in some respects like a notch, and (iii) cases where a large volume of material is affected and a simple stress concentration, or $\mathrm{K}_{\mathrm{T}}$ approach may be employed. 
More recent asymptotic approaches have been developed by Dini et al. [43, 44] and are concerned primarily with bounded asymptotics. The approach does not relate so directly to crack solutions, but provides a potential means of correlating fretting contacts under different geometry and loading conditions. Consider, for example, the 'flat and rounded' contact depicted in Fig. 11a, which gives a similar contact pressure to that in the dovetail (Fig. 3). Far away from the radius at the corner, and provided we are also far from the other edge of the contact the arguments of [39] may be applied to conclude that the presence of the radius has very little effect and the pressure will be similar to that for a flat punch with sharp corners (Equation (4)). Very close to the edge of contact, the presence of the radius will be the dominant feature and the pressure distribution will be bounded. It can be shown further that, asymptotically, the pressure will be Hertzian at the edge of contact so that

$$
p(r)=K_{I}^{*} \sqrt{r}
$$

where $r$ is again measured from the edge of contact and $K_{I}^{*}$ is a parameter describing the intensity of normal loading. These 'inner' and 'outer' asymptotes for pressure are shown schematically in Fig. 11b. The surrounding stress field may be characterised using Muskhelishvili potential methods [45]. The relevant potential is

$$
\Phi(z)=\frac{K_{I}^{*}}{2} \sqrt{z}
$$

A similar approach may be adopted for tangential loading, where it is possible to deal with the case of partial slip. If the stick/slip boundary is located at a distance $d$ from the contact edge, the shear traction may be described by

$$
\begin{array}{lr}
q(r)=\frac{2 K_{I I}^{*}}{d} \sqrt{r} & 0<r<d \\
q(r)=\frac{2 K_{I I}^{*}(\sqrt{2}-\sqrt{r-d})}{d} & r>d
\end{array}
$$

where $K_{I I}^{*}$ describes the intensity of tangential loading. $K_{I}^{*}, K_{I I}^{*}$ and $d$ are related by

$$
\frac{K_{I I}^{*}}{f K_{I}^{*}}=\frac{d}{2}
$$

It will be noted that $K_{I}^{*}, K_{I I}^{*}$ are dimensionally different.

\section{Short crack arrest}

The asymptotic approaches outlined above generally offer a means of correlating one contact with another, under different geometric and loading conditions. Thus, it should be possible to characterise a contact in a component and to carry out fretting fatigue experiments under an identical stress state. This offers an extremely useful means of reducing the complexity of the experiments required and experimentally characterising initiation lives. However, fretting fatigue experiments are still likely to be required and a more ambitious goal would be to seek to predict fretting fatigue performance from materials parameters obtained in other types of experiment (for example the plain fatigue strength or long crack threshold $\Delta \mathrm{K}$. There seems little hope of characterising initiation in this way, since the surface finish is likely to play an extremely important role at these length scales and detailed description of the required parameters is likely to prove extremely challenging. At a slightly larger length scale, however, surface roughness effects are likely to be less dominant. We have already remarked 
that for a crack to initiate and grow, the stress field needs to be high and sustained over a reasonable distance. This observation can be formalised by appealing to the concepts of short crack arrest. The general approach was first suggested by Nowell and Araújo [46] and independently by Chan et al. [47]. The method is based on the Kitagawa-Takahshi diagram [48], expressing the crack propagation threshold as a function of crack length for small cracks. Let us assume that there exists a long crack threshold $\Delta \mathrm{K}$, denoted by $\Delta \mathrm{K}_{0}$. Long cracks will propagate only if $\Delta \mathrm{K}$ is above this value, i.e.

$$
\Delta K>\Delta K_{0}
$$

On the other hand, short cracks may be observed to initiate and grow if the applied stress range exceeds the fatigue limit

$$
\Delta \sigma>\Delta \sigma_{f l}
$$

This equation may be conveniently expressed in terms of the stress intensity factor as

$$
\Delta K>Y \Delta \sigma_{f l} \sqrt{\pi \alpha}
$$

where $Y$ is the usual geometric factor associated with the stress intensity factor and $\alpha$ is the crack length. These two equations (10) and (12) may be plotted on a diagram of $\Delta \mathrm{K}$ vs $\alpha$, as shown in normalised form in Fig. 12. The intersection of the curves occurs at a crack length $\alpha_{0}$ given by

$$
\alpha_{0}=\frac{1}{\pi}\left(\frac{\Delta K_{0}}{Y \Delta \sigma_{f l}}\right)^{2}
$$

which for the case of $Y=1$ corresponds to the well-known El Haddad dimension (El Haddad et al. [49]). This allows us to write equation (12) as

$$
\Delta K>\Delta K_{0} \sqrt{\frac{\alpha}{\alpha_{0}}}
$$

The region in Fig. 12 below both of the curves may be regarded as a 'safe' region of no crack propagation, whereas the region above either curve is likely to result in crack initiation and growth.

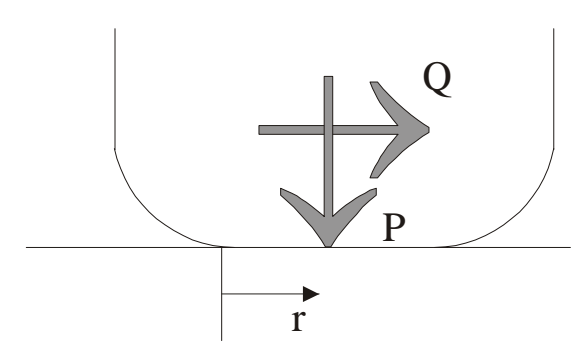

(a)

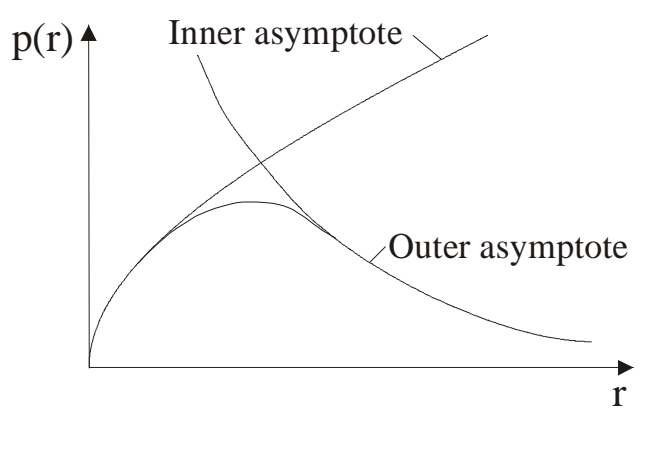

(b)

FIGURE 11. (a) Flat and rounded contact geometry, (b) schematic diagram of inner and outer asymptotes for pressure. 
This approach may now be used to predict a fretting fatigue limit as follows: the variation of stress intensity factor with crack length for a given situation is first obtained. This is then plotted on the K-T diagram in the manner shown schematically in Fig. 13. Generally, the resulting plot will be of two forms (i) the SIF curve will be always above the threshold (curve A) in which case failure can be expected, or (ii) the SIF curve falls below the threshold (curve $\mathrm{B}$ ) in which case a growing crack will arrest. The limiting case may be found by determining the load corresponding to a curve of type $\mathrm{C}$, which just touches the threshold.

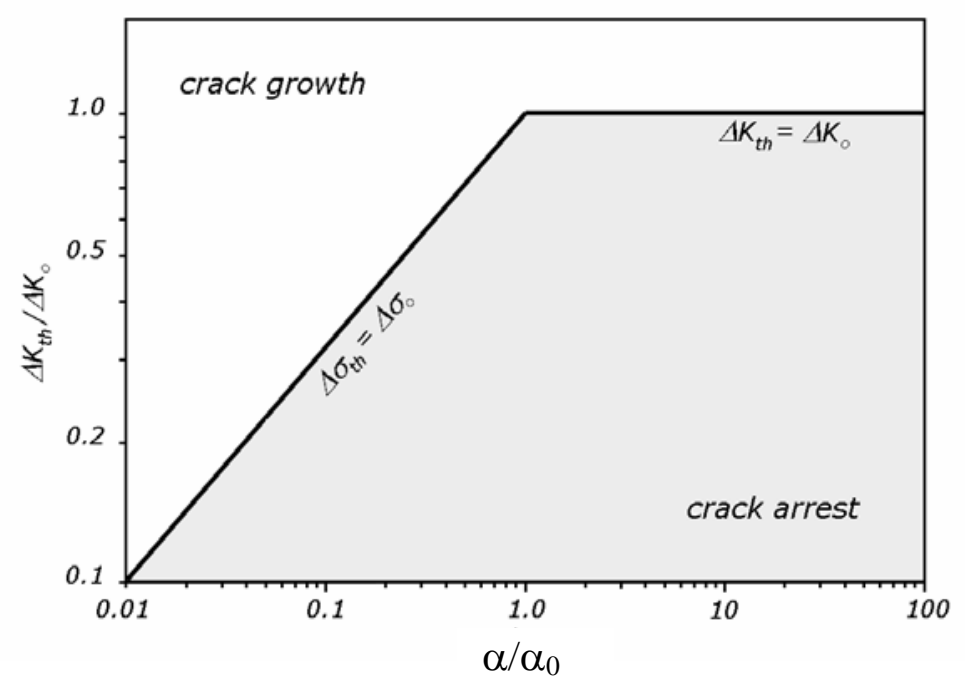

FIGURE 12 - The Kitagawa-Takahashi Diagram

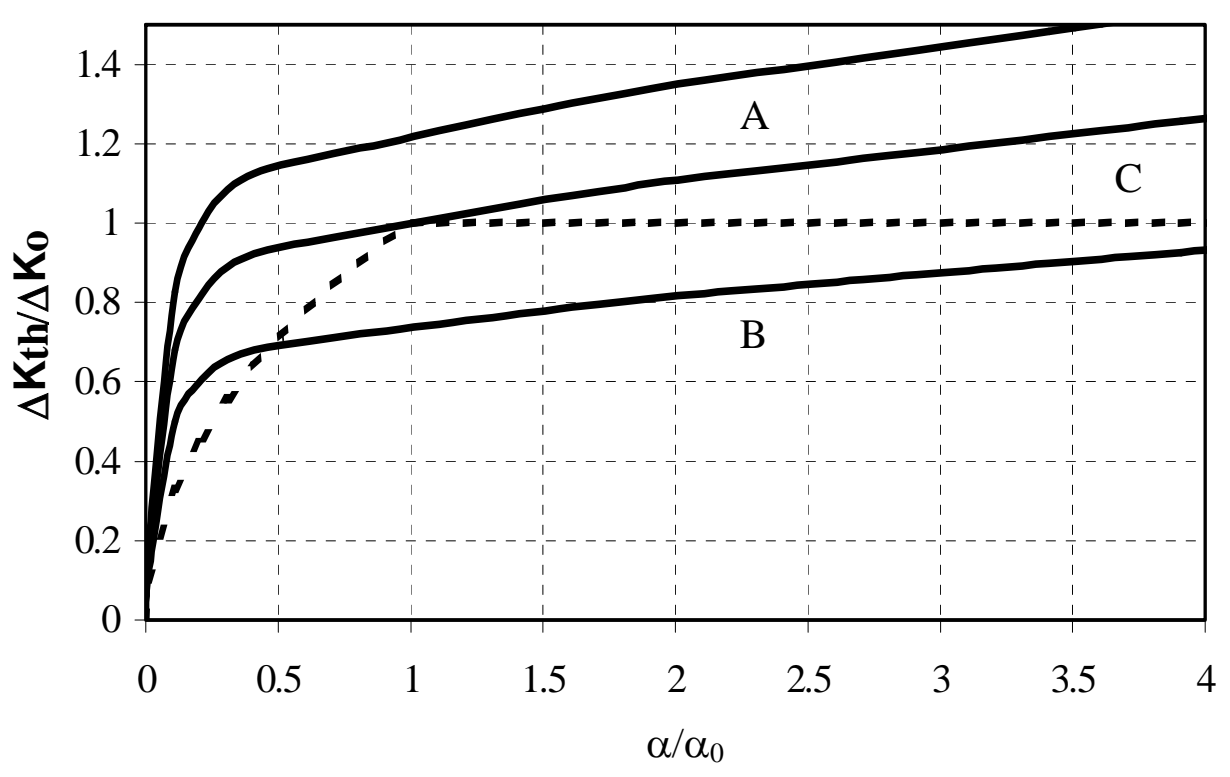

FIGURE 13 - Schematic crack growth trajectories in the Kitagawa-Takahashi Diagram

This approach may be used with some success to predict the fretting fatigue strength for a range of configurations, as depicted in Fig. 14 for a sample case of flat and rounded pads against a Ti-6Al-4V specimen. It will be seen that the size effect is clearly predicted by this approach. In many cases, as for the results shown here, the predictions are rather similar to those obtained using a notch analogy and the 'point' method at an appropriate depth beneath the contact (c.f. Fig. 10). The short crack approach is, in principle, more attractive since it relies only on standard material parameters obtained from plain fatigue experiments. It should 
be noted, however, that there may be some uncertainty in measured values of $\Delta \mathrm{K}_{0}$ and $\Delta \sigma_{\mathrm{fl}}$ since neither are entirely straightforward to determine. Similarly, the choice of $Y$ in equation (13) is not without difficulty and values may range from 0.67 for a semi-circular crack to 1.1215 for an edge crack in a two-dimensional geometry. The fact that these parameters are combined and then raised to the power of two in equation (13) may lead to significant uncertainties in $\alpha_{0}$ in a practical case, so that although it has a clear physical basis it might be better regarded as a fitting parameter, in which case some of the attractiveness of the approach is lost. We should also note in passing that an alternative definition for the threshold may be developed based on the short crack arguments advanced by El Haddad et al. in [49]. Traditionally, the El-Haddad approach is seen as increasing the crack driving force by increasing the effective crack length by $\alpha_{0}$ so that

$$
\Delta K_{e f f}=Y \Delta \sigma \sqrt{\pi\left(\alpha+\alpha_{0}\right)}
$$

Implicit in this approach is the assumption that the threshold remains constant. An alternative means of considering the modification is to regard the crack driving force as constant

$$
\Delta K_{e f f}=Y \Delta \sigma \sqrt{\pi \alpha}
$$

but that the threshold is reduced so that

$$
\Delta K_{t h}=\Delta K_{0} \sqrt{\frac{\alpha}{\alpha+\alpha_{0}}}
$$

It will be seen that this gives a single expression for the threshold which is asymptotic to equations (10) and (14) for long and short cracks respectively, whilst allowing for some interaction between the two regimes, so that at $\alpha=\alpha_{0}$ equation (16) predicts a threshold which is $70 \%$ of that given by the intersection of (10) and (14).

\section{Conclusions}

The problem of fretting fatigue is very complex, involving high stress gradients, nonproportional multiaxial loading, and surface finish effects which are difficult to characterise. However, significant progress has been made in understanding the phenomenon over the past decade. An important foundation for this work has been the increased standardisation of test techniques and a recognition of the importance of monitoring a wide range of parameters during the course of the experiment. The improved reliability of experimental data has removed some of the obstacles present in developing predictive methods. A number of attempts have been made to apply multiaxial initiation parameters to fretting fatigue, but it is now recognised that the stress gradients present are frequently very high and it is therefore necessary to average the parameters or the underlying stresses over a representative volume in order to capture the experimentally observed size effect.

In characterising the initiation problem a number of parallels have been drawn between fretting fatigue and notch fatigue. In retrospect it perhaps seems surprising that these were not recognised earlier. However, for many practical cases, stress gradients in fretting fatigue are much higher than in notches which are present as design features so that it is far from straightforward to compare fretting and notch fatigue results. A more promising approach appears to be the use of asymptotic methods to characterise the stress fields at the edge of contact, which is frequently the site of crack initiation. Initially these methods dealt with singular stress fields corresponding to the 'flat punch' problem, but modifications have now been made to deal with practical geometries and the use of bounded asymptotics to 
characterise initiation conditions appears to provide a useful means of correlating initiation conditions between apparently dissimilar fretting situations.

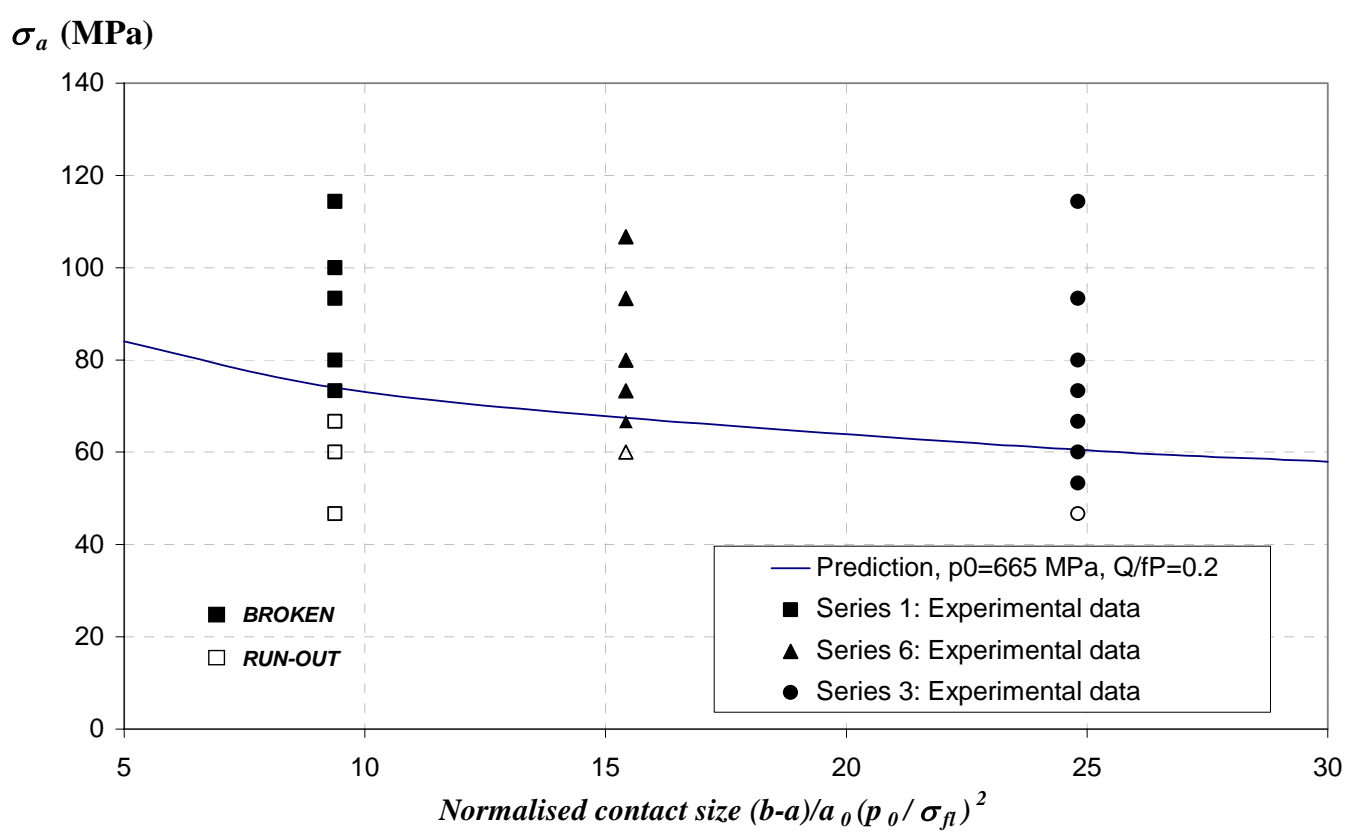

FIGURE 14. Predictions of the short crack arrest method compared to fretting fatigue test results for flat and rounded pads on Ti-6Al-4V specimens.

Long crack propagation in fretting fatigue is, in principle, no different to that of plain fatigue, although some complications remain, such as the presence of variable R-ratio and non-proportional loading. Short crack propagation is particularly important in the fretting situation, since the length scales of the contact stress field and microstructurally short cracks frequently coincide. Short crack arrest methods have been developed which are able to predict the arrest of fretting cracks from plain fatigue data, specifically the fatigue limit and the long crack threshold $\Delta \mathrm{K}$. These approaches show considerable promise as a means of predicting the fretting fatigue limit in practical situations. Looking into the future, a number of areas look increasingly important. The importance of microstructure offers scope for investigation since microstructural dimensions are often similar to the characteristic dimension of the stress field. The use of conventional continuum mechanics may not therefore always be appropriate. Similarly, most surfaces will contain residual stress fields, but these are frequently ignored in analyses of fretting fatigue. Improved surface treatment processes such as laser shock peening will increasingly be used in fretting situations and there will therefore be a need to predict fretting performance in the presence of residual stress.

\section{References}

1. Eden, E.M., Rose, W.N. and Cunningham, F.L., Proc. I.Mech.E., vol. 4, 139, 1911.

2. Hutson, A.L., Ashbaugh, N.E., and Nicholas, T. In Fretting Fatigue: advances in the basic understanding and applications, edited by S.E. Kinyon, D.W. Hoeppner, and Y. Mutoh, ASTM, West Conshohocken, USA, 2003, 307-322.

3. Tomlinson, G.A., Thorpe P.L., and Gough, H.J., Proc. I.Mech.E., vol. 141 , 223-249, 1939. 
4. Warlow-Davies, E.J., Proc. I.Mech.E., vol. 146 , 33-38, 1941.

5. Maxwell, W.W., Dudley, B.R., Cleary, A.B., Richards, J., and Shaw, J., Proc. I.Mech.E, vol. 182, 89-108, 1967.

6. Hirakawa, K., Toyama, K., and Kubota, M., Int. Jnl Fatigue, vol. 20, 135-144, 1998.

7. Burns, C., (Ed.) Proceedings, $7^{\text {th }}$ National Turbine Engine High Cycle Fatigue (HCF) Conference (on CD), Universal Technology Corporation, Dayton, Ohio, 2002.

8. Nowell, D., In Progress in Structural Mechanics, edited by J. Morton and F. Paris, University of Seville, 2000, 61-72.

9. Xi, N.S., Zhong, P.D.; Huang, H.Q., Yan, H., Tao, C.H., Engineering Failure Analysis, vol. 7, 385, 2000.

10. Fenner, A.J., and Field, J.E., Proc. N.E. Coast Institute of Engineers and Shipbuilders, vol. $76,183,1960$.

11. Nishioka, K., and Hirakawa, K., Bull. JSME, vol. 15 , 135-144, 1972.

12. Hertz, H., Jnl. reine und angewandte Mathematik, vol. 92, 156-171, 1882.

13. Mindlin, R.D., Jnl. App. Mech., vol. 16 , 259-268, 1949.

14. Bramhall, R., Studies in Fretting Fatigue, D.Phil. thesis, University of Oxford, 1973.

15. Hills, D.A., Nowell, D., and O'Connor, J.J., Wear, vol. 125, 129-156, 1988.

16. Szolwinski, M.P., and Farris, T.N., Wear, Vol. 221, 24-36, 1998.

17. Mugadu, A., Hills, D.A., and Nowell, D., Wear, vol. 252, 475-483, 2002.

18. Ruiz, C., Boddington, P.H.B., and Chen, K.C., Experimental Mechanics, vol. 24, 208-217, 1984.

19. Papanikos, P., and Meguid, S.A., Fatigue Fract. Engng Mats Struct., vol. 17, 539-550, 1994.

20. Conner, B.P., and Nicholas, T., ASME Jnl Eng Mats and Tech., in press, 2004.

21. Johnson, K.L., Contact Mechanics, Cambridge University Press, 1984.

22. Fellows, L.J., Nowell, D., and Hills, D.A., Wear, vol. 185, 235-238, 1995.

23. Hills, D.A., and Nowell, D., Int. Jnl Fatigue, vol. 23, 747-748, 2001.

24. Nowell, D., An Analysis of Fretting Fatigue, D.Phil. thesis, University of Oxford, 1988.

25. Nishioka, K., and Hirakawa, K., Bull. JSME, vol. 12, 692-697, 1969.

26. Vingsbo, O., and Söderberg, D., Wear, vol. 126, 131-147, 1988.

27. Waterhouse, R.B., Int. Mater. Reviews, vol. 37, 77-97, 1992.

28. Ciavarella, M., Dini, D., and Demelio, G.P., Proceedings of the $10^{\text {th }}$ International Conference on Fatigue, Honolulu, December 2001, Edited by K. Ravi-Chandar, B.L. Karihaloo, T.Kishi, R.O. Ritchie, A.T. Yokobori, Jr, and T. Yokobori, Pergamon, 2001.

29. Neu, R.W., Pape, J.A., and Swalla, D.R., in Fretting Fatigue: Current Technology and Practices, ASTM STP 1367, edited by D.W. Hoeppner, V. Chandrasekaran, and C.B. Elliott, ASTM, West Conshohocken, PA, 2000, 369-388.

30. Fatemi, A., and Socie, D., Fatigue Fract. Engng Mats Struct., vol. 11, 145-165, 1988. 
31. Smith, R.N., Watson, P., and Topper, T.H., Jnl of Mats, JMLSA, vol. 5. 767-778, 1970.

32. Araújo, J.A., and Nowell, D., Int. Jnl Fatigue, vol. 24, 763-775, 2002.

33. Fouvry, S., Kapsa, P., and Vincent, L., in Fretting Fatigue: Advances in the Basic Understanding and Applications, ASTM STP 1425, edited by S.E. Kinyon, D.W. Hoeppner, and Y. Mutoh, ASTM, West Conshohocken, PA, 2003, 17-32.

34. Dang Van, K., Griveau, B., and Message, O., in Biaxial and Multiaxial Fatigue, edited by M.W. Brown and K.J. Miller, Mech. Eng Pubs, London, 1989, 479-496.

35. Peridas, G., Korsunksy, A.M., and Hills, D.A., Jnl Strain Analysis, vol. 38, 201-206, 2003.

36. Murthy, H., Farris, T.N., and Slavik, D.C., in Materials Science Research International, STP 1, JSMS, Kyoto, 2001, 200-207,

37. Nowell, D., and Dini, D., Tribology International, vol. 36, 71-78, 2003.

38. Taylor, D., Fatigue and Fracture of Engineering Materials and Structures, vol. 24, 215224, 2001.

39. Giannakopoulos, A.E., Lindley, T.C., and Suresh, S., Acta Mater., vol. 46, 2955-2968, 1998.

40. Giannakopoulos, A.E., Lindley, T.C., and Suresh, S., Fatigue and Fracture of Engineering Materials and Structures, vol. 23, 561-571, 2000.

41. Ciavarella, M., Fatigue and Fracture of Engineering Materials and Structures, vol. 26, 1159-1170, 2003.

42. Atzori, B., and Lazzarin, P., Int. Jnl Fracture, vol. 107, 1-8, 2001.

43. Dini, D., Studies in Fretting Fatigue with Particular Application to Almost Complete contacts, D.Phil. Thesis, University of Oxford, 2004.

44. Dini, D. and Hills, D.A., Int. Jnl of Solids and Structures, under review, 2004.

45. Muskhelishvili, N.I. Some Basic Problems of the Mathematical Theory of Elasticity, English translation by J.R.M. Radok, Noordhoff, 1953.

46. Nowell, D., and Araújo, J.A., in Small fatigue cracks: Mechanics, Mechanisms, and Applications, edited by K.S Ravichandran, R.O. Ritchie, and Y.Murakami, Elsevier, Oxford, 1999, 361-372.

47. Chan, K.S., Lee, Y-D., Davidson, D.L., and Hudak, S.J., Jr, Int. Jnl Fracture, vol. 112, 299-330, 2001.

48. Kitagawa, H., \& Takahashi, S., in Proceedings of the 2nd International Conference on Mechanical Behaviour of Materials, American Society for Metals, 1976, 627-631.

49. El Haddad, M.H., Smith, K.N., and Topper, T.H., Jnl of Eng Mats and Tech., vol. 101, 42-46, 1979.

\section{Acknowledgements}

The financial support of Rolls-Royce plc and the UK Department of Trade and Industry for some of the work described is gratefully acknowledged. I would also like to acknowledge the helpful discussions I have held with my colleagues Professor David Hills and Mr Daniele Dini during the course of preparing this paper. 